\title{
Industrial applications of sheet stamping simulation using new finite element models
}

\author{
J.Rojek, J. Jovicevic, E. Oñate \\ International Center for Numerical Methods in Engineering \\ Univ. Politécnica de Cataluña \\ Gran Capitán, s/n, 08034 Barcelona,Spain
}

\begin{abstract}
The paper is aimed to present industrial applications of sheet stamping simulation using new finite element formulations developed in the International Center for Numerical Methods in Engineering in Barcelona. Theoretical formulation is briefly reviewed. Both continuum and shell elements have been considered. The new shell elements developed are based on a geometrically exact shell model treating the shell as one-director Cosserat surface. The formulation of the continuum elements employs the multiplicative decomposition of the deformation gradient tensor into its elastic and plastic parts. The new finite element models have been implemented in the in-house explicit dynamic code STAMPACK. A number of practical problems of sheet metalforming have been solved with the program. Some of the problems, namely stamping of a kitchen sink, hydraulic forming of an aeronautical part and stamping of a food can, have been presented in the paper. The examples give an idea of practical information that can be obtained from the computer simulation of a forming process. The results confirm a good behaviour of the formulation and program used in the industrial applications.
\end{abstract}

Keywords: sheet stamping, industrial applications, finite element simulation, explicit dynamic analysis,

\section{Introduction}

Finite element simulation of stamping processes has been successfully applied to the analysis of real parts, it is still however an interesting problem for investigators aiming at the development of enhanced finite element models yielding accurate results for complex problems while being at the same time computationally effective. In recent years new element formulations for the sheet forming simulation have been developed in the International Center for $\mathrm{Nu}$ merical Methods in Engineering and implemented in the numerical code STAMPACK based on the explicit dynamic approach [6]. The results of practical industrial applications presented in this paper show a good behaviour of the new finite element models.

\section{New finite element models developed}

\subsection{Continuum element formulation}

In the numerical simulation of sheet stamping the part deformed can be discretised with shell or continuum elements. Both approaches have their well known advantages and disadvantages. In our work both types of elements have been considered. The formulation of the continuum elements used [3] employs the multiplicative decomposition of the deformation gradient tensor $\mathbf{F}$ into its elastic and plastic parts, $\mathbf{F}^{\mathbf{e}}$ and $\mathbf{F}^{\mathrm{p}}$, respectively

$\mathbf{F}=\mathbf{F}^{\mathrm{e}} \mathbf{F}^{\mathrm{P}}$

Using this decomposition the elastic Finger tensor is calculated as

$\mathbf{b}^{\mathrm{e}-1}=\mathbf{F}^{\mathrm{e}-\mathrm{T}} \mathbf{F}^{\mathrm{e}-1}$

This in turn is used in the calculation of the Almansi strain tensor

$\mathbf{e}^{\mathbf{e}}=\frac{1}{2}\left(\mathbf{g}-\mathbf{b}^{\mathbf{e}-1}\right)$

where $\mathbf{g}$ is the spatial metric tensor. Hyperelasticity is assumed in the constitutive model and the Cauchy stresses $\boldsymbol{\sigma}$ are recovered from the free energy function $\psi$ written in terms of Almansi elastic strains $\mathbf{e}^{\mathbf{e}}$

$\boldsymbol{\sigma}=\frac{\partial \psi\left(\mathbf{e}^{\mathbf{e}}\right)}{\partial \mathbf{e}^{\mathbf{e}}}$

The potential is adopted in the form

$\psi^{\mathrm{e}}=\frac{1}{2} \lambda \operatorname{tr}\left(\mathbf{e}^{\mathrm{e}}\right)^{2}+\mu\left(\mathbf{e}^{\mathrm{e}}: \mathbf{e}^{\mathrm{e}}\right)$

where $\lambda$ and $\mu$ are the Lamé constants, which is valid with the assumption of small elastic deformations fully justified for metals. Material yielding is treated using the Von Mises criterion with isotropic nonlinear hardening. 
In the numerical implementation a 4-node $2 \mathrm{D}$ element and 8-node hexahedral 3D element have been employed. Both elements are based on the linear approximation of the displacement field and a constant pressure is used to avoid the volumetric locking.

\subsection{Shell element formulation}

Continuum elements offer the possibility to use a more exact 3D description of the deformation and allow us to consider better two face contact conditions. However due to a greater numerical efficiency a shell model of the blank is more often used in the simulation. A family of shell elements based on the nonlinear shell theory developed by Simo and co-workers $[7,8]$ have been derived [2], as well as a new shell element without rotational degrees of freedom has been worked out using a finite volume-like approach $[1,5]$. The former shell formulation is based on the so-called geometrically exact shell model treating the shell as one-director Cosserat surface. It is assumed that any configuration of the shell can be described completely in terms of:

- the mid-surface of the shell, defined by the mapping $\varphi$ : $\mathcal{A} \rightarrow R^{3}$

- the director field defined by the mapping $\mathrm{t}: \mathcal{A} \rightarrow S^{2}$ where $\mathcal{A}$ is the set of reference points $\xi=\left(\xi^{1}, \xi^{2}\right), \xi \in R^{2}$, and $S^{2}$ is the unit sphere defined as $S^{2}:=\left\{t \in R^{3}|||t| \mid=\right.$ 1) With these definitions the loda of the shell $\mathrm{x}\left(\mathrm{x} \in R^{3}\right)$ can be giver by $\mathbf{x}\left(\xi^{1}, \xi^{2}, \xi\right)=\varphi\left(\xi^{1}, \xi^{2}\right)+\xi \mathbf{t}\left(\xi^{1}, \xi^{2}\right)$ . $\xi)=\varphi\left(\xi^{1}, \xi^{2}\right)+\xi t\left(\xi^{1}\right.$, convective membrane, bendit
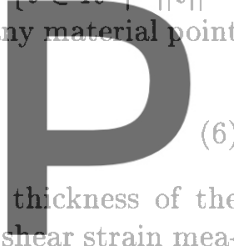
sures, $\varepsilon_{\alpha \beta}, \rho_{\alpha \beta}$ and $\delta_{\alpha}$, respectively, are defined at the shell

Registerterfopp ffee at https//www.scipedia.com to

$\varepsilon_{\alpha \beta}=\frac{1}{2}\left(\varphi, \alpha \cdot \varphi_{, \beta}-\varphi_{, \alpha}^{0} \cdot \varphi_{, \beta}^{0}\right)$

$\rho_{\alpha \beta}=\boldsymbol{\varphi}_{, \alpha} \cdot \mathbf{t}_{, \beta}-\mathbf{t}_{, \alpha}^{0} \cdot \boldsymbol{\varphi}_{, \beta}^{0}$

$\delta_{\alpha}=\varphi_{, \alpha} \cdot \mathbf{t}-\mathbf{t}_{, \alpha}^{0} \cdot \varphi^{0}$

where $\alpha, \beta=1,2$ and index " 0 " denotes the initial configuration. Having been calculated in the convective base the strains are transformed into the Cartesian base defined at the initial configuration as the Total Lagrangian formulation is used in the description of large deformations. Two alternative constitutive models have been employed, the stress resultant one with the Ilyushin yield criterion and the other one make use of continuum constitutive law with the integration across the shell thickness.

Using the formulation presented above a four-node isoparametric quadrilateral and six-node triangular element have been derived. In the quadrilateral element both the shell mid-surface and the director field are interpolated with bilinear functions, in the triangular element quadratic functions are used. In both cases a mixed formulation with assumed shear strain have been used to avoid shear locking.

The shell elements presented above have been found quite efficient in the analysis of sheet stamping problems, nevertheless still more efficient solution have been looked for. A new shell element without rotational degrees of freedom, called Basic Shell Triangle (BST) has been derived [5]. The BST element is composed of the standard Constant Strain Triangle membrane element and a new triangular plate element with a single degree of freedom per node [5]. The plate element formulation is based on the following formula obtained with finite volume-like approach

$\iint_{A_{\mathrm{s}}} \boldsymbol{\kappa} \mathrm{d} A=\int_{\Gamma_{\mathrm{s}}} \mathrm{T} \nabla w \mathrm{~d} \Gamma$

where

$\boldsymbol{\kappa}=\left\{\kappa_{x}, \kappa_{y}, \kappa_{x y}\right\}^{T}=\left\{-\frac{\partial^{2} w}{\partial x^{2}},-\frac{\partial^{2} w}{\partial y^{2}},-2 \frac{\partial^{2} w}{\partial x \partial y}\right\}^{T}$

$\mathbf{T}=\left[\begin{array}{cc}n_{x} & 0 \\ 0 & n_{y} \\ n_{y} & n_{x}\end{array}\right]$

$\nabla=\left\{\frac{\partial}{\partial x}, \frac{\partial}{\partial y}\right\}^{T}$

with $A_{\mathrm{S}}$ being an arbitrary subdomain of a plate (shell), $\Gamma_{s}$ its boundary, $x$ and $y$ Cartesian coordinates in the plane of the plate, $w$ deflection and $\mathbf{n}=\left\{n_{x}, n_{y}\right\}^{\mathrm{T}}$ a unit vector

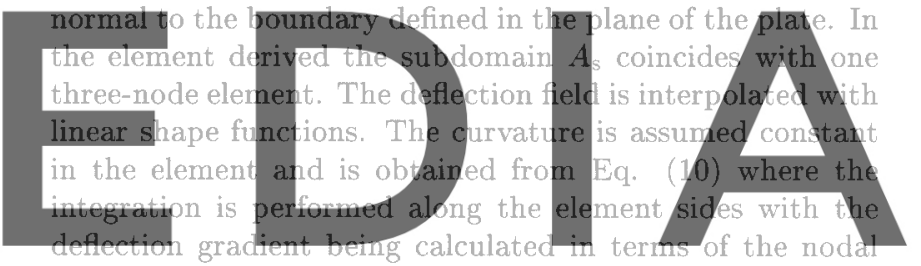

deflections of the adjacent elements. The element is adopted

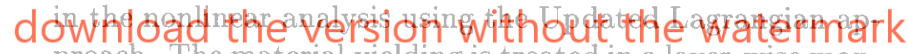
proach. The material yielding is treated in a layer-wise man-

ner assuming isothropic or anisothropic plastic properties.

The shell element library is complemented with axisymmetric shell elements and strip elements for 2D plane deformation problems. In this case a standard two-node Reissner-Mindlin and Kirchhoff elements have been implemented $[4,10]$.

\section{Industrial problems of sheet metalforming}

\subsection{Simulation of stamping of a kitchen sink}

The process of deep drawing of a kitchen sink from a stainless steel sheet $0.7 \mathrm{~mm}$ thick has been studied using the finite element model of a half of the structure. The nonuniform mesh of 5312 8-node hexahedral continuum elements has been used in the discretisation of the sheet. The blankholder has been considered deformable and has been modelled with 120 elements the same as those used in the sheet discretisation. The selection of the continuum elements for the model of the sheet and the deformable model of the blankholder have been motivated by a better consideration of an important role played by the nonuniform distribution of the pressure between the blankholder and sheet which was observed in the real process. Continuum elements have enabled us to capture the influence of the 


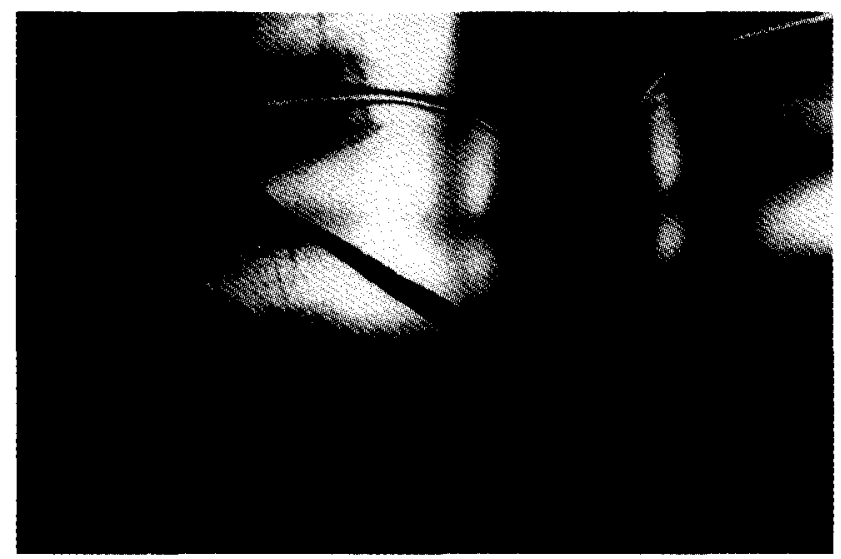

Fig. 1: Kitchen sink - a real part with breakage

change of sheet thickness on the contact pressure distribution.

The part has been found very difficult to stamp. The drawing depth of $140 \mathrm{~mm}$ has been found impossible to be obtained in one stroke, so a two stage stamping process have been adopted in the workshop. This has increased production costs the more that a manual lubricating at some zones of the sheet was necessary before each of the two strokes since a uniform lubrication did notensure the proper stamping process even with two stage forming. The flow of naterial was controlled with nonuniform lubrication as well as with ponuniform distribution of the blankholder pressyre. The use of drawbeads was impossible because of the quality requirements for the product. With all these means the breakage occurred in the part if on stage operation was tried, usually at the depth of drawing of about $120 \mathrm{~mm}$. Register for free at haterved / whown in.Fig. llia.com to stamping for the cases of uniform and nonuniform lubrication. The results in the form of the deformed shape with the thickness distribution for the case of uniform lubrication at the punch stroke of $130 \mathrm{~mm}$ are presented in Fig. 2. There can be seen a significant thinning of the sheet on the punch corner coinciding with the place of breakage of a real part. The formability of the part is verified using the Forming Limit Diagram (Fig. 3). Forming Limit Curve has been calculated from the theoretical Stören-Rice formula [9]. There can be seen some points above the FLC on the diagram, which means that the state of breakage has been reached at these places of the sink. The zone represented by the points above the FLC on the right hand side of the FLD coincides with the zone of the fracture shown in Fig. 1. The points above the FLC on the left side of the FLD represent the zone which is on the minimum die radius, where the stress state is dominated by the bending and the maximum deformation occurs on the surface only. In some experiments the fracture occurred in this place as well. Summing up we can state that the simulation reproduced well the real process of stamping a kitchen sink.

\subsection{Simulation of hydraulic forming with diaphragm}

Sheet metal forming has traditionally involved three rigid piece tools. Some new forming techniques which do not use the traditional tooling have been developed. In many cases they can be used instead of conventional methods yielding technological and tangible benefits. One of these methods is hydraulic forming with diaphragm that employs hydraulic action of oil on the sheet placed on the rigid punch. The sheet is separated from the liquid medium
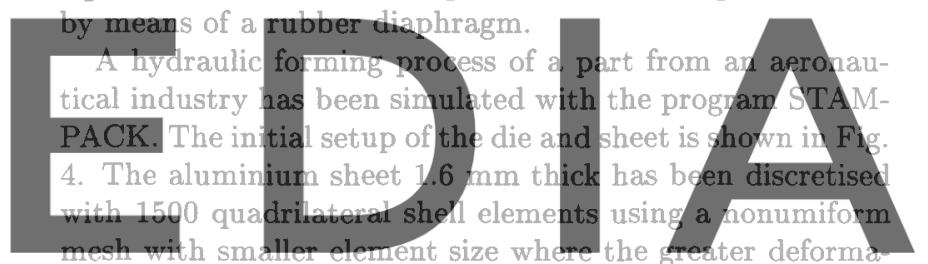

tion gradients have been expected. One of the features of

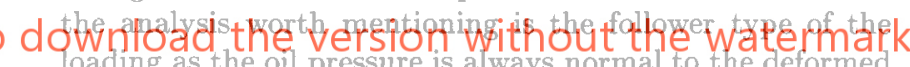
configuration. The material properties have been considered rate independent and inertial effects have been considered of minor importance so we applied the total pressure of 80 $\mathrm{MPa}$ as a step loading.

A comparison between the numerical results for the sheet deformations and the results obtained in the real process is

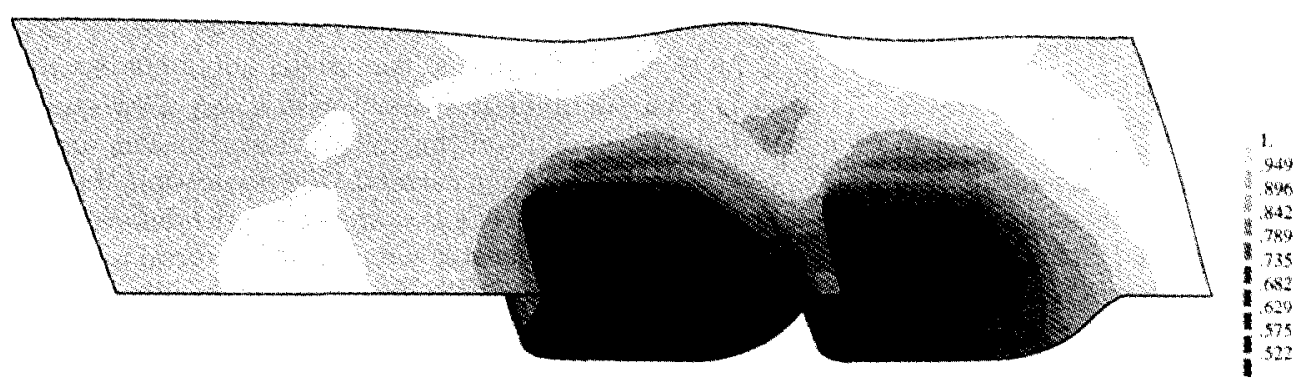

Fig. 2: Deformed shape of the kitchen sink with the distribution of the thickness normalised with respect to the initial thickness 


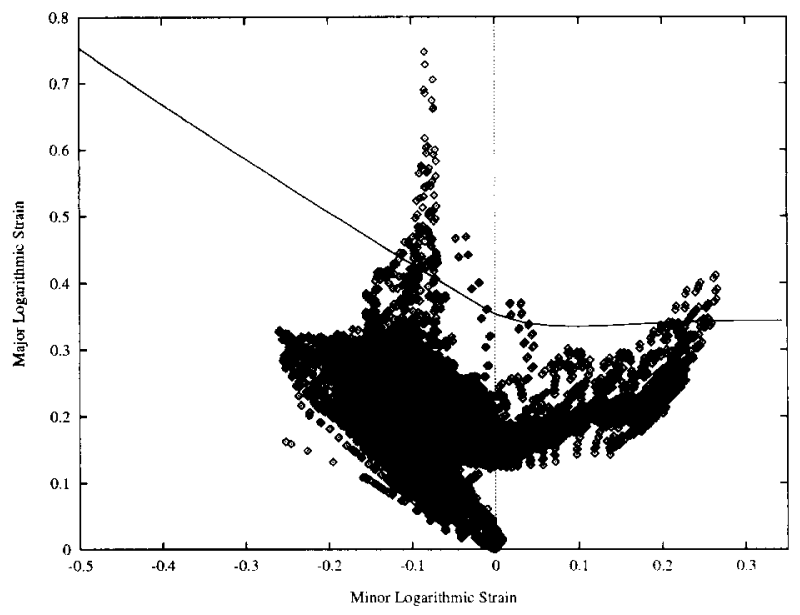

Fig. 3: Forming Limit Diagram for the kitchen sink

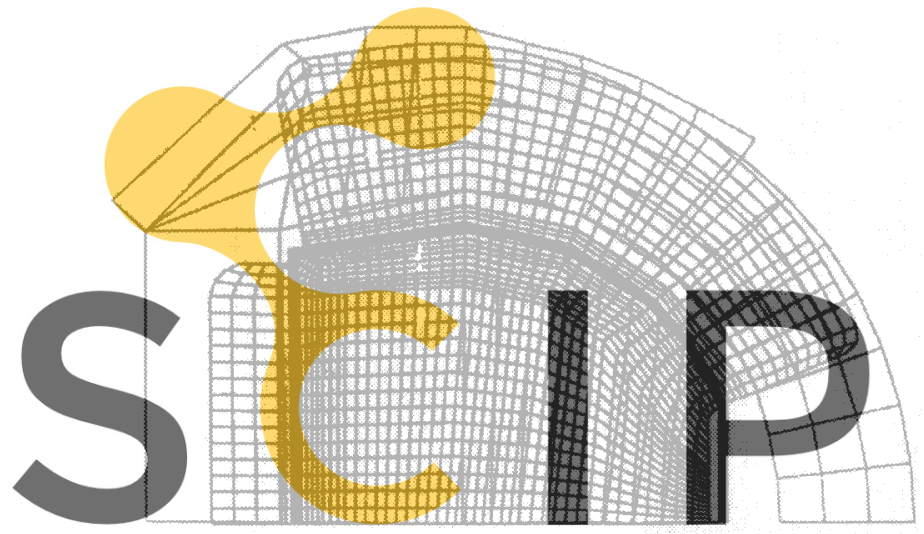

Register for free at https//www.scipedia.com to do Fig. 4: Initial setup of the die and sheet

shown in Fig. 5. Excellent agreement between the results compared has been found. It can be seen that the simulation reproduces accurately an important defect due to local buckling in the circular flange region. There is a localised large wrinkle here. This example shows the possibility of verification of a manufacturing process involving hydraulic forming technique at the stage of the process design.

\subsection{Simulation of deep drawing of an axisymmetric food can}

Deep drawing process of an axisymmetric food can from aluminium sheet has been analysed. The initial diameter of the flange rim is $116.5 \mathrm{~mm}$ and the initial thickness of the sheet is $0.22 \mathrm{~mm}$. The initial and deformed configurations of the tools and sheet at different stages of forming are shown in Fig. 6. The sheet was discretised with 2064 node continuum axisymmetric elements using one element over thickness. The problem was analyzed for different friction coefficients $\mu=0,0.05$ and 0.15 . The draw-in values of the sheet flange obtained in the analysis were compared with the values measured in the real process. The values
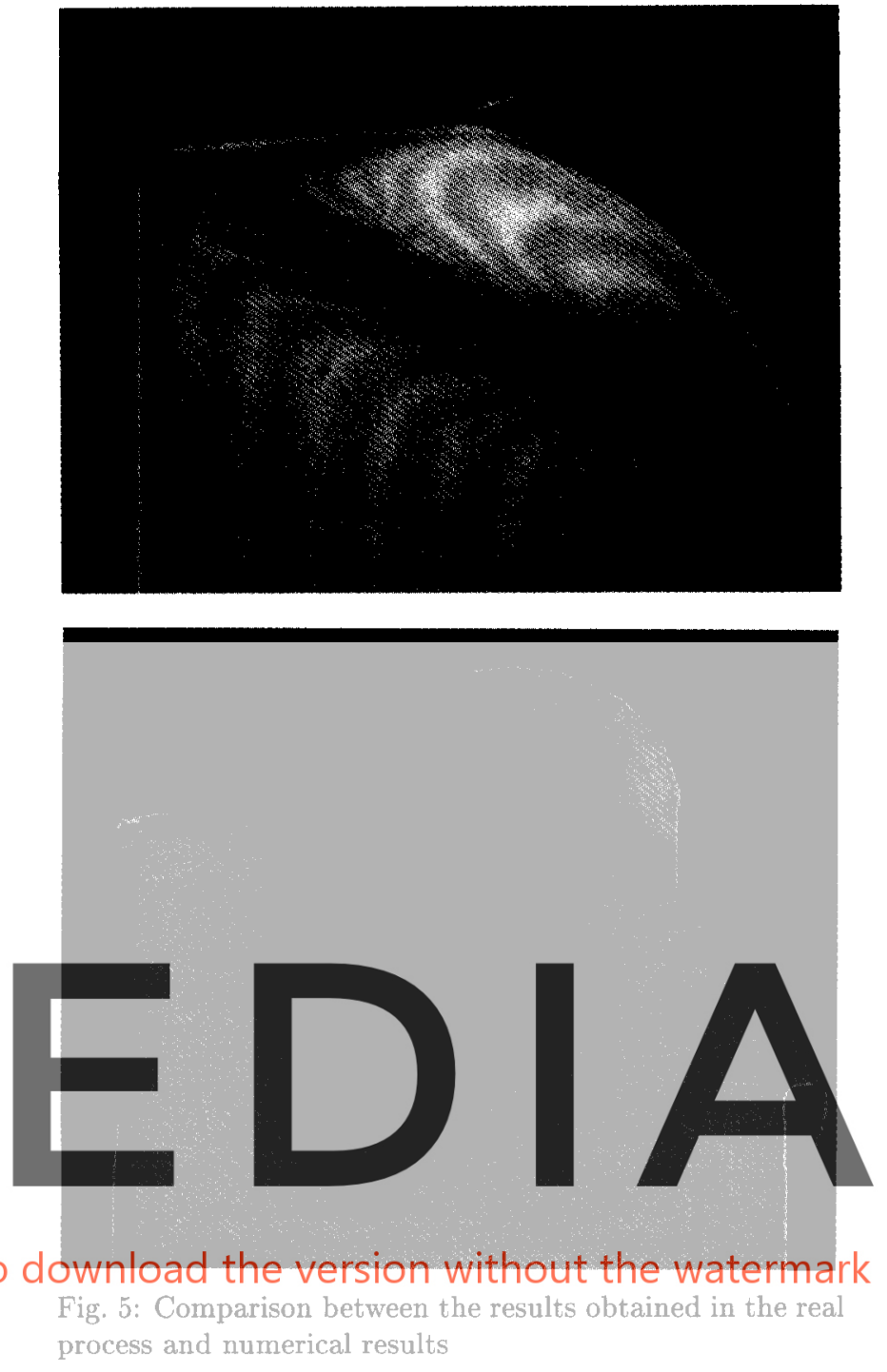

obtained $19.2 \mathrm{~mm}$ for $\mu=0,18.7 \mathrm{~mm}$ for $\mu=0.05$ and $16.9 \mathrm{~mm}$ for $\mu=0.15$ are in a very good agreement with the average real value $17.3 \mathrm{~mm}$. The problem was analysed later using the discretisation with 103 axisymmetric shell elements. Three layers were used in each element to consider plastification of the section of the shell. The results obtained are practically the same as those obtained with continuum model. The advantage of the shell model is evident when compared to the continuum one as the shell model enables us to use much greater time steps and a coarser mesh.

This examples provides us with a very interesting application of the results of the numerical simulation, namely use of the results to design correctly the decoration of the can. The decoration of the can is designed for the deformed part (Fig. 8) however the sheet is painted before forming (Fig. 7). The painting pattern of the sheet is traditionally obtained by an trial-and-error procedure not always with satisfactory results. This process has been automated. The specialised software has been developed to transform the painting from the flat sheet to the deformed part or in the opposite way according to the deformation of the sheet obtained from the finite element simulation. Fig. 7 presents the painting of the 
sheet obtained with a traditional method and Fig. 8 shows the decoration on the deformed can predicted with the new methodology after performing transformation of the image from Fig. 7. Some possible defects of the decoration can be perceived. This shows that the method worked out can be used to verify the correctness of the painting pattern.

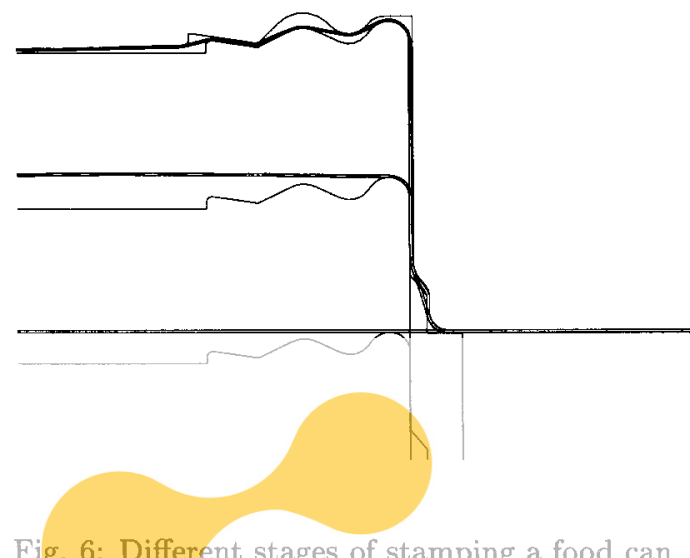

Fig. 6: Different stages of stamping a food can

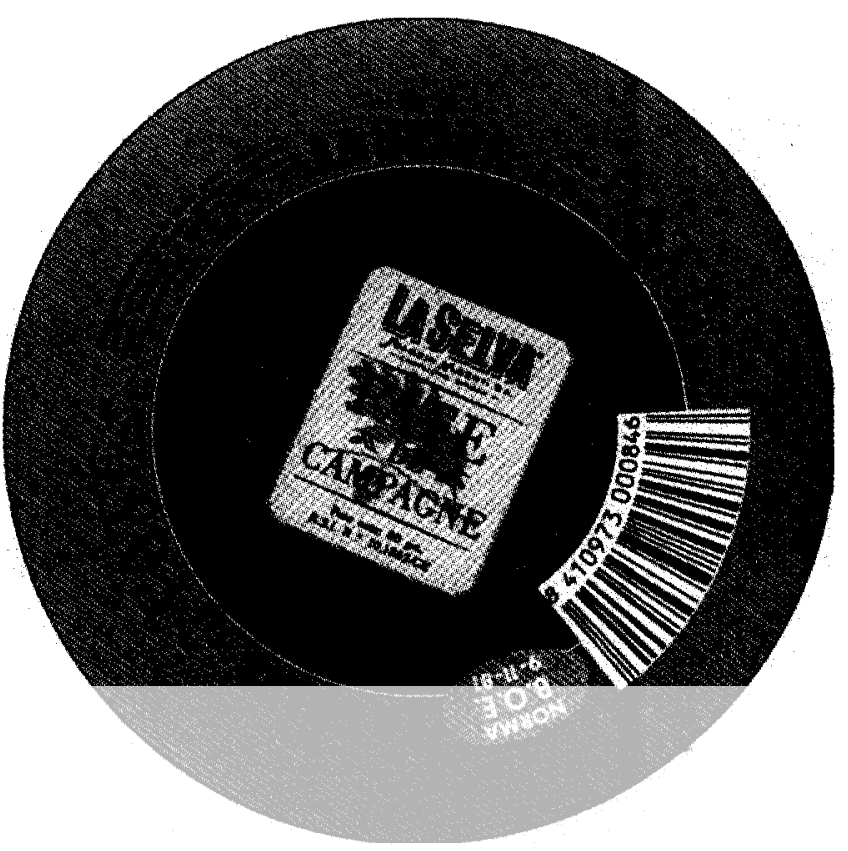

Fig. 7: Painting of a flat sheet

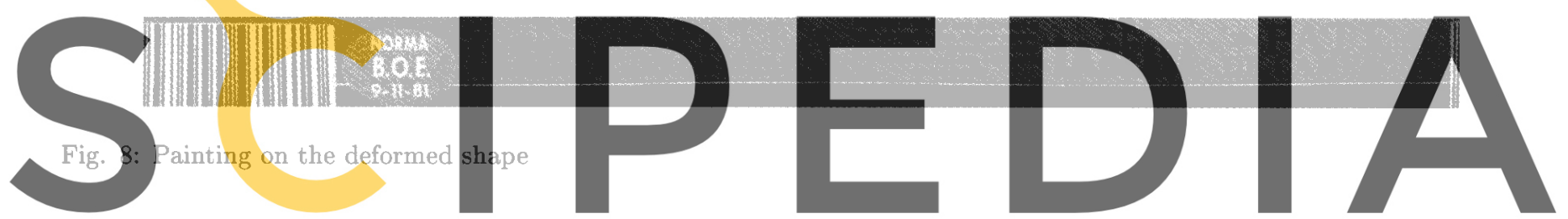

Registerofolufire ratmattes//www.scipedia.com to downdoadhthe versionatvithioditidhewatermark

The results of numerical simulation of the industrial sheet metalforming processes presented show a good behaviour of the new finite element models developed and implemented in the program STAMPACK. The examples show possible application of the finite element simulation in the verification of the makeability of the part thanks to possibility of predicting part defects such as breakage and wrinkling. The numerical simulation can be used to correct or optimise the forming process. It can also be underscored that the practical applications included reflect a great interest in the use of numerical simulation existing in small companies of the sheet stamping sector.

\section{References}

[1] P. Cendoya. New Finite Elements for Elastoplastic Dynamic Analysis. PhD thesis, Universitat Politecnica de Catalunya, Barcelona, to be published in 1996. (in Spanish).

[2] F. Flores and E. Oñate. New shell elements for nonlinear structural analysis. Technical report, CIMNE, Barcelona, 1993.

[3] C. García Garino. A Numerical Model for the Analysis of Large Elasto-plastic Deformations of Solids.
Barcelona, 1993. (in Spanish).

[4] M.E. Honnor. Non-linear Finite Element Analysis of Axisymmetric Shells Applied to Sheet Metal Forming. $\mathrm{PhD}$ thesis, University of Wales, Swansea, 1985.

[5] E. Oñate and M. Cervera. A general procedure for deriving thin plate bending elements with one degree of freedom per node. CIMNE, Barcelona, 1993.

[6] E. Oñate, J. Rojek, and C. García Garino. NUMISTAMP: a research project for assesment of finite element mo dels for stamping processes. Journal of $\mathrm{Ma}$ terials Processing Technology, 50(1-4):17-38, 1995.

[7] J.C. Simo and J.G. Kennedy. On a stress resultant geometrically exact shell model. Part V: Nonlinear Plasticity. Formulations and integration algorithms. Comput. Meth. Appl. Mech. Eng., 96:133-170, 1992.

[8] J.C. Simo, M.S. Rifai, and D.D. Fox. On a stress resultant geometrically exact shell model. Part IV: Variable thickness shells with through-the-thickness stretching. Comput. Meth. Appl. Mech. Eng., 81:91-126, 1990.

[9] S. Stören, J.R. Rice, Localized necking in thin sheets, $J$. Mech. Phys. Solids, 123, 1986.

[10] O.C. Zienkiewicz. The Finite Element Method in Engineering Science. McGraw-Hill, second edition, 1971. 